\title{
Analysis of the prognostic significance and potential mechanisms of IncRNAs associated with m6A methylation in papillary thyroid carcinoma
}

\section{Yinde Huang}

The First Affiliated Hospital of China Medical University

\section{Xin Li}

The First Affiliated Hospital of China Medical University: The First Hospital of China Medical University

\section{Wenbin Chen}

The First Affiliated Hospital of China Medical University

\section{Yuzhen He}

The First Affiliated Hospital of China Medical University

\section{Song Wu}

The First Affiliated Hospital of China Medical University

\section{Xinyang Li}

The First Affiliated Hospital of China Medical University

\section{Bingchen Hou}

The First Affiliated Hospital of China Medical University

\section{Shiyue Wang}

The First Affiliated Hospital of China Medical University

\section{Yuchen He}

The First Affiliated Hospital of China Medical University

\section{Han Jiang}

The First Affiliated Hospital of China Medical University

\section{Yu Lun}

The First Affiliated Hospital of China Medical University

Jian Zhang ( $\boldsymbol{D}$ jianzhang@cmu.edu.cn )

the First Affiliated Hospital of China Medical University https://orcid.org/0000-0003-4448-0774

\section{Research Article}

Keywords: m6A, Papillary thyroid carcinoma, IncRNA, prognostic biomarker, PD-L1

Posted Date: August 16th, 2021

DOI: https://doi.org/10.21203/rs.3.rs-807935/v2 
License: (c) (i) This work is licensed under a Creative Commons Attribution 4.0 International License. Read Full License

Version of Record: A version of this preprint was published at International Immunopharmacology on December 1st, 2021. See the published version at https://doi.org/10.1016/j.intimp.2021.108286. 


\section{Abstract}

Background : m6A methylation-related long non-coding RNAs (IncRNAs) play a significant role in the progression of various tumors and can be used as prognostic markers. However, whether m6A-related IncRNAs also play the same function as prognostic markers in papillary thyroid carcinoma (PTC) remains unclear.

Methods : Consensus cluster analysis was performed to divide PTC samples obtained from The Cancer Genome Atlas database into two clusters according to the expression of m6A-related IncRNAs. Then, the least absolute shrinkage and selection operator (LASSO) regression analysis was performed to create and verify a prognostic model. Furthermore, the relationship among risk scores, clusters, programmed death-ligand 1 (PD-L1), tumor microenvironment (TME), clinicopathological characteristics, immune infiltration, immune checkpoint, and tumor mutation burden (TMB) was analyzed. In addition, a nomogram was created, and subsequently, the drug sensitivity of IncRNAs in the prognostic model was analyzed. Finally, the relationship between these IncRNAs and prognosis in pan-cancer was investigated.

Results: The prognosis, RAS, BRAF, M, and TME were found to be different in two clusters. The prognostic model included three IncRNAs: PSMG3-AS1, BHLHE40-AS1, and AC016747.3 . The risk score was associated with clusters, PD-L1, tumor microenvironment, clinicopathological characteristics, immune cell infiltration, immune checkpoint, and TMB, and thus, risk score was confirmed as useful prognostic indicators. Differentially expressed IncRNAs are involved in many malignancies and can be identified as cancer prognostic makers.

Conclusion : According to our research, we can regard m6A-related IncRNAs involved in the procession of PTC as a biomarker of PFS for PTC patients, and pan-cancer.

\section{Introduction}

The incidence of thyroid cancer, the most common endocrine cancer, has been continuously increasing in recent decades [1, 2]. Papillary thyroid carcinoma (PTC) is the most common type of thyroid cancer with an excellent prognosis [3-5]. However, approximately $30 \%$ of patients with PTC have a poor prognosis due to local recurrence or distant metastasis [6, 7]. Individuals with poorer prognoses should be crucially identified, and the mechanism of PTC development should be deeply understood.

Post-transcription modifications have become a significant regulator of many physiological processes and disease development, which attracts researchers' interest in cancer studies [8]. A previous study has shown that $\mathrm{N}(6)$-methyladenosine (m6A) is involved in the most common internal (non-cap) alteration of the messenger RNA (mRNA) in all higher eukaryotes, which are essential for cell viability and development [9]. There are three types of m6A regulators: the methyltransferase complex (MTC) often known as "writers" that catalyzes m6A; demethylase, commonly known as "eraser" that removes the m6A; and the RNA reader protein identifies m6A, attaches to the RNA, and performs the necessary activities. In cancer etiology and progression, writers, erasers, and readers are crosslinked [10, 11]. 
Researchers show great interest in long non-coding RNAs(IncRNAs) after understanding their functions. LncRNAs, the non-coding transcripts, are involved in at least 200 nucleotides [12]. However, their functions are still mostly unclear. LncRNAs may function as mRNA expression control via translational regulation, histone changes, or posttranscriptional regulation [13]. They can also impact numerous tumor cell characteristics (survival, proliferation, and migration) by gene control [12]. Aberrant IncRNA expression is involved in tumor aggressiveness and procession in various malignancies, including PTC. At this point, researchers have concluded that m6A, a necessary part of the complex IncRNA process, functions on tumor development [14]. However, the mechanism and prognostic value of m6A-related IncRNAs in PTC remains unknown.

Transcriptome data of PTC were obtained from a public source (TCGA-THCA database) and divided into two groups according to the consensus cluster analysis based on the expression level of m6A-related IncRNAs. To further accurately predict the progression-free survival (PFS) of patients with PTC, the prognostic model of m6A-related IncRNAs was developed and verified. The relationship among, programmed death-ligand 1 (PD-L1), tumor microenvironment (TME), risk score, clinicopathological characteristics, immune cell infiltration, tumor mutation burden (TMB), and clusters were also analyzed. Subsequently, to explore the predicted probability of the PFS in patients with PTC, a nomogram was drawn, and the drug sensitivity of three IncRNAs was analyzed in the prognostic model. Finally, the relationship among three IncRNAs and the prognosis in pan-cancer was also analyzed.

\section{Materials And Methods}

\section{Data collections}

Relevant genetic alterations and pathways that influence the tumor genesis, progression, differentiation, and metastasis [15] can be investigated because of a tremendous amount of genomic and clinical data available in the Cancer Genome Atlas (TCGA). The TCGA-THCA dataset contains 58 adjacent nontumorous tissues $(\mathrm{N})$ and 510 patients with PTC $(\mathrm{T})$ and 487 with simple nucleotide variation data (VarScan), which are used to calculate the TMB of patients with PTC. The University of California Santa Cruz (UCSC) genome database (https://xenabrowser.net; accessed 12 July 2021) provided the clinical data of 501 patients with PTC $[16,17]$. Patients with incomplete follow-up information were excluded.

To broaden the scope of our study, 23 traditional regulators were included based on previous studies: writers (METTL3, METTL14, METTL16, WTAP, VIRMA [KIA1499], ZC3H13, RBM15, and RBM15B), readers (YTHDC1, YTHDC2, YTHDF1, YTHDF2, YTHDF3, HNRNPC, FMR1, LRPPRC, HNRNPA2B1, IGFBP1, IGFBP2, IGFBP3, and $R B M X)$, and erasers (FTO and $A L K B H 5)$ [18-20].

\section{Screening out IncRNAs associated with m6A methylation}

After extracting expressions of $23 \mathrm{m6A}$ regulators from the TCGA-THCA dataset, co-expression analysis between m6A regulators and m6A-related IncRNAs is performed using the "limma" package. Based on the "|correlation coefficient| of $\geq 0.5$ " and " $p$-value of $<0.001$," co-expression analysis was conducted and 
regulators of m6A methylation-related IncRNAs were selected using the Pearson correlation test. The expression information of the co-expression network for m6A RNA methylation-related IncRNA was also achieved in the "igraph" R package. m6A-related IncRNAs associated with the PFS of patients with PTC were screened using univariate COX progression analysis, and IncRNAs with $p<0.01$ were included in the follow-up study.

\section{Consensus Clustering Analysis}

In the TCGA-THCA cohort, patients with PTC were divided according to clusters and examined the biological features of m6A-related IncRNAs using the R package "ConsensusClusterPlus." Then, the PFS of clusters were estimated using the Kaplan-Meier analysis. The probable functionalities and downstream access of two clusters were observed using the Estimation of Stromal and Immune cells in Malignant Tumor tissues using Expression data (ESTIMATE) to evaluate the composition of the TME including the estimate score (stromal-immune comprehensive score), immune score (immune content), stromal score (stromal content). The R package "corrplot" was used to explore the correlation between the expression levels of PD-L1 and m6A-related IncRNAs.

\section{Construction and validation of the prognostic model}

After evenly dividing samples collected from TCGA-THCA into training and testing datasets, the least absolute shrinkage and selection operator (LASSO) regression analysis method was performed using the "glmnet" package [21] in the R4.1.0 to develop the prognostic model of m6A-related IncRNAs for PTC samples in the training dataset. The risk score of the LASSO regression model can be calculated as follows [22]:

$$
\text { Risk score }=\sum_{i=1}^{n} \beta_{i} * E_{i}
$$

where $\beta_{i}$ represented the IncRNA coefficient and $E_{i}$ represented the IncRNA expression. The risk score was calculated using this method in the training, testing, and TCGA-THCA datasets. Three datasets were divided into the high- and low-risk groups according to the median risk score. The area under the curve (AUC) was used to analyze the predictive power of signatures using the receiver operating characteristic (ROC) curves. The "stats" R package with the "prcomp" function was used to show principal component analysis (PCA) for the IncRNA expression. t-Distributed Stochastic Neighbor Embedding (t-SNE) was also performed using the "Rtsne" R package to explore variation from different groups in terms of their distribution. Heatmaps were drawn to display the distribution of clinicopathological characteristics between the high- and low-risk groups.

\section{Analysis of the value of prognostic models}

To assess the usefulness of risk score as independent prognostic variables, Cox regression models were used with other clinical characteristics (age [ $\geq 55$ vs. $<55$ years], gender [male vs. female], American Joint 
Committee on Cancer [AJCC] stage [III+IV vs. I+II], AJCC M [M1 vs. M0], AJCC N [N1 vs. N0], AJCC T [T3+4 vs. T1+2], BRAF [mutated vs. wild-type), RAS [mutated vs. wild-type], and risk score (high vs. low]). A stratification study was conducted to determine whether risk score retained their capacity in analyzing PFS among different subgroups according to the order stratified analysis based on clinicopathological characteristics, which further evaluates the prognostic capability. A heatmap was created using the $R$ package "pheatmap" that depicted the correlation between clinicopathological variables and groups. Given the significance of checkpoint inhibitor-based immunotherapies, differences in 47 immune checkpoint expressions were explored further between the two subgroups, and the connection among these IncRNAs was shown (47 immune checkpoints are provided in Supplementary List 1). Subsequently, we investigated the correlation among risk scores, immune cells, or TMB using the Pearson correlation analysis.

\section{Sensitivity Analysis of Chemotherapy}

In the cancer research community, a database, known as Cellminer, was developed to determine the integration and analysis of molecular and pharmacological data for the $\mathrm{NCl}-60$ malignant cell lines collected by the United States Developmental Therapeutics Program. More than 100,000 chemical compounds and natural items will be screened by the National Cancer Institute (since 1990) (https://discover.nci.nih.gov/cellminer/home.do). The Pearson correlation analysis was used to evaluate the connection between prognostic IncRNAs expression and drug sensitivity. The correlation study was used to explore the effectiveness of 263 medicines that have been authorized by the Food and Drug Administration or are currently undergoing clinical studies.

\section{Prognosis-related IncRNA's pan-cancer value promotion}

The Gene Expression Profiling Interactive Analysis 2 (GEPIA2) (http://gepia2.cancer-pku.cn/\#index), which can integrate the TCGA and Genotype-Tissue Expression Project (GTEx) database, was used to analyze the pan-cancer value about prognostic-related IncRNAs [23]. Expression levels of three prognostic-related IncRNAs were first investigated in 33 tumors and normal tissues ( $p$-value $<0.05, \| \log F C \mid$ $>0.1$, differential methods: limma). We also explored the relationship between IncRNA and the prognosis of 33 tumors.

\section{Statistical analysis}

The Kaplan-Meier survival analysis (PFS considered as the end-point) was performed using the "survival" package in R. The chi-square test was performed to explore differences in clinical parameters of the highand low-risk groups in the training, testing, and overall TCGA-THCA datasets. The Pearson's correlation analysis was performed to assess related variables. A pair of independent t-test samples was used to compare the results in the Mann-Whitney test to explore differences between high- and low - risk samples. A log-rank test was used to calculate the $\mathrm{p}$-values for PFS. Statistical analyses were performed and visualized in $\mathrm{R}$ version 4.1.0, IBM SPSS Statistics for Windows version 25.0 (IBM Corp., Armonk, NY, USA), and GraphPad Prism version 8.0 (GraphPad Software Inc., La Jolla, CA, USA). 


\section{Results}

\section{Analyzing the consensus cluster based on median expression levels of m6A-related IncRNAs in patients with PTC}

We firstly found that 630 IncRNAs co-expressed with 15 m6A methylation regulators and then used them to visualize a co-expression network (Supplementary Figure 1). Red nodes show the m6A RNA methylation regulators, and blue nodes display m6A-related IncRNAs.

To further investigate the relationship between IncRNAs and the PFS of patients with PTC, we found prognostic-related IncRNAs (Figure 2A, B) using the univariate COX analysis and collected 21 IncRNAs (AC010168.2, CAPN10-DT, PSMG3-AS1, AL662844.4, AC010761.3, AC093752.3, LMNTD2-AS1, AL031705.1, CD44-AS1, U73166.1, BHLHE40-AS1, MZF1-AS1, AL360181.2, AC138956.1, AL359878.1, AC002128.1, AC084125.2, AC139795.2, AC016747.3, AL355987.4, and AC138956.2) for subsequent studies.

Based on the expression of 21 m6A-related IncRNAs, the TCGA-THCA cohort was divided into distinct clusters using the "ConsensusClusterPlus" R package (Figure 2C, D, E). Regarding 2 as the consensus matrix's k-value, the least crossover was obtained from all PTC samples. The difference in PFS was explored between two clusters using the Kaplan-Meier technique. PFS was significantly longer in cluster 1 (PFS, $p=0.005$ ) as compared to cluster 2 (Figure 2F). After comparing the clinicopathological characteristics of two clusters and analyzing their connection, RAS, BRAF, and AJCC M stage were all significantly related to our cluster analysis (Figure $\mathbf{2 G}$ ). The percentage of the 22 immune cell types in two clusters was analyzed using the CIBERSORT. CD8 T cells, CD4 memory actived T cells, follicular helper T cells, macrophages M1, and activated dendritic cells were significantly different between the two clusters (Figure 2H). The TME was examined using the ESTIMATE, stromal, and immune scores and found significant differences in the two groups (Figure 2l, J, K). Considering the important function of PDL1 in tumor treatment, a correlation was observed among the PD-L1 and 21 m6A-related IncRNAs (Figure 2L) and the expression of PD-L1 between PTC samples and normal tissues in clusters 1 and 2 (Supplementary Figure 2A, B). The expression of PD-L1 was negatively correlated with 18 IncRNAs. Statistically significant changes in PD-L1 expression were observed between tumor and healthy control. However, compared to cluster 2, expression levels of PD-L1 expression were higher in cluster 1.

\section{Identification and verification of the prognostic model of m6A-related IncRNAs}

Next, the effectiveness of the prognostic model was evaluated. After randomly dividing the entire TCGATHCA dataset into two groups (training dataset $[n=252]$ and testing dataset $[n=249]$ ), a prognostic model was obtained in the training dataset using the LASSO regression analysis based on the expression of 21 IncRNAs (Supplementary Figure 3A, B). A total of three IncRNAs (PMSG3-AS1, BHL HE40-AS1, and AC016747.3) were included in the prognostic model. The risk score of patients in the training, testing, and entire TCGA-THCA dataset were calculated by the prognostic model. The following prognostic model formula was used: risk score $=(0.264992826432626 *$ PSMG3-AS1 expression level $)+$ 
$\left(-0.777201837590316{ }^{\star} B H L H E 40-A S 1\right.$ expression level) $+\left(1.34421059388075^{\star} A C 016747.3\right.$ expression level). According to the median risk score, patients in all three datasets were divided into high- and lowrisk groups. The associations among the risk score, prognostic status, and expressive signatures of three m6A-related IncRNAs in the training, testing, and entire TCGA-THCA cohorts are shown (Supplementary Figure 4A-I). The PFS between the two groups was further analyzed, with significant differences, whether in the training dataset $(p=0.004$, Figure $3 \mathrm{~A})$, the testing dataset $(p=0.001$, Figure $3 \mathrm{~B})$, or the entire TCGATHCA dataset $(p<0.001$, Figure $3 \mathrm{C})$. As shown in the time-dependent ROC curves, significant differences were found between the high- and low-risk groups. AUC analyses for 1-, 2-, 3-year PFS prediction according to the IncRNA signature were $0.747,0.626$, and 0.722 in the training dataset (Figure 3D); 0.709, 0.651 , and 0.771 in the testing dataset (Figure 3E); $0.733,0.634$, and 0.739 in the entire TCGA-THCA dataset (Figure 3F). PCA and t-SNE analyses displayed that patient in the three datasets were dispersed in two methods (Figure 3G-L). Thus, the risk score is relatively accurate in determining the PFS of patients with PTC because it is greatly distinct among patients.

Univariate and multivariate Cox analyses were performed to examine the association between the risk score and PFS. A risk score was found as a risk factor for PFS in all three datasets, as demonstrated by the univariate cox analysis results (training dataset: hazard ratio $[\mathrm{HR}]=2.767,95 \%$ confidence interval $[\mathrm{Cl}]=1.891-4.050, p<0.001$, Figure 3M; testing dataset: $\mathrm{HR}=6.817,95 \% \mathrm{Cl}=2.375-19.561, p<0.001$, Figure $3 \mathrm{~N}$; entire TCGA-TCHA dataset: $\mathrm{HR}=3.147,95 \% \mathrm{Cl}=2.260-4.383, p<0.001$, Figure 30). In the multivariate Cox regression analysis, the risk score remained a significant predictor of PFS even considering the effects of the other factors (training dataset: $\mathrm{HR}=2.849,95 \% \mathrm{Cl}=1.702-4.771, p<0.001$, Figure 3P; testing dataset: $\mathrm{HR}=40.147,95 \% \mathrm{Cl}=3.020-533.628, p=0.005$, Figure 3Q; entire TCGA-THCA dataset: $\mathrm{HR}=2.458$, $95 \% \mathrm{Cl}=1.593-3.794, p<0.001$, Figure 3R).

\section{Stratification analysis based on clinicopathological characteristics}

The prognostic profile associated with m6A-mediated IncRNAs might be used to predict PFS in both highand low-risk groups. A stratified analysis on clinicopathological variables showed appropriate results,

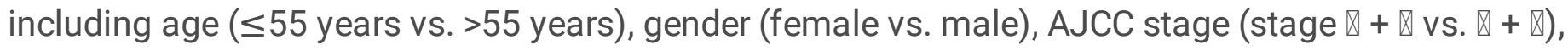
AJCC T stage ( $T \nabla+\nabla$ vs. $T \nabla+\varangle)$, AJCC M stage (M0 vs. M1), AJCC N stage (N0 vs. N1), BRAF (wild-type vs. mutated), and RAS (wild-type vs. mutated) ( $p<0.05$, Figure 4). The Kaplan-Meier method of survival analysis has been performed, and results have shown that the high-risk group has a shorter PFS than the low-risk group in the number of clinical strata, except for patients aged $<55$ years, and patients with RAS mutation (Figure 4A). This additional proof confirmed our hypothesis on its significance.

\section{The correlation between risk score and clinicopathology characteristics, cluster analysis results, or immune scores}

When comparing high- with low-risk samples from the TCGA-THCA dataset with clinicopathological factors, immune scores, and cluster analysis results, expression levels of PSMG3-AS1 and AC016747.3 were found to be higher in the high-risk group (Figure 5A), indicating high-risk IncRNAs. Conversely, the expression level of BHLHE4O-AS1 in low-risk samples was higher than in high-risk samples, indicating a 
low-risk IncRNA. A higher-risk value can be observed in the RAS-mutated (Figure 5B), BRAF wild-type (Figure 5C), NO (Figure 5D), age $\geq 55$ years (Figure 5E), ImmuneScore low (Figure 5F), and cluster 2 (Figure 5G). Then, we found differences in the expression levels of 30 immune checkpoints between highand low-risk samples (Figure $5 \mathrm{H}$ ).

\section{Correlation between risk score with immunocytes and tumor mutation burden and prognostic nomogram}

After performing the CIBERSORT analysis, Pearson correlation was used to analyze the correlation between risk scores and 22 immune cells. A positive correlation can be observed between regulating $T$ cells (Tregs) and risk score (Figure 6C), whereas the correlation between resting dendritic cell (Figure 6A) or resting mast cell (Figure 6B) and risk scores was negative. A positive correlation also be observed between TMB and risk score (Figure 6D). To quantitatively predict the patient's prognosis, a nomogram was created based on clinicopathological characteristics and risk scores to accurately predict the patient's PFS (Figure 6E).

\section{Sensitivity analysis of chemotherapy}

Two prognosis-related IncRNAs (PSMG3-AS1, BHLHE40-AS1) were found to correspond to 11 drugs (Figure 7). As the PSMG3-AS1 expression increases, the sensitivity of cancer cells to these drugs (mitomycin C, mitoxantrone, tegafur, idarubicin, gemcitabine, fulvestrant, and teniposide) increases. As the PSMG3-AS1 expression increases, the resistance of cancer cells to the drug (bosutinib) increases. The sensitivity of cancer cells to epirubicin, mitoxantrone, and doxorubicin and their resistance to allopurinol increase while increasing the BHLHE4O-AS1 expression levels. Remarkably, as the expression of these two IncRNAs increases, the sensitivity of cancer cells to mitoxantrone increases (Figure $7 C, H$ ).

\section{Prognosis-related IncRNA's pan-cancer value promotion}

We found that these three IncRNAs are differentially expressed in most tumors (Figure 8A, C, E). The results show that the overall survival rate is significantly different with the increasing PSMG3AS1 and BHLHE40-AS1 expression levels (Figure 8B, D). A significant difference in disease-free survival rate was observed between patients with low- and high expressions of AC016747.3 (Figure 8F).

\section{Discussion}

The role of post-transcriptional modification in life activities has increasingly received attention [8]. Among the many RNA modifications, m6A is the most common type of modification and is involved in the occurrence and development of different tumors [14, 24]. Some studies have reported that METTL3 inhibits the progression of thyroid papillary carcinoma by affecting neutrophil infiltration through $\mathrm{m} 6 \mathrm{~A} / \mathrm{c}-$ Rel/IL-8 [25] and regulating m6A methylation on TCF1 [26]. Another study has found that the protein content of FTO correlated with lymph node metastasis and tumor grade is an independent prognostic factor of thyroid cancer grade [27]. Some studies have shown that m6A-methylation-related regulators can predict the prognosis of patients with PTC using bioinformatics methods [28-30]. Therefore, the 
formation and prognosis of patients with PTC can be evaluated with m6A methylation. The function of IncRNAs in different cancers has greatly increased the interest of researchers. Thus, studies have claimed that $\mathrm{m} 6 \mathrm{~A}$ is a key step in the IncRNA process that plays a role in the pathological process of tumors [31], and we can infer the prognosis of patients through the expression of m6A-methylation-related IncRNAs, such as pancreatic adenocarcinoma [32], ovarian cancer [33], hepatocellular carcinoma [34], triplenegative breast cancer [35], primary head and neck squamous cell carcinoma [14], kidney renal clear cell carcinoma [36], metastatic skin cutaneous melanoma [37], and lower-grade glioma [38]. No studies have shown the possibility of predicting the prognosis of patients with PTC by m6A-related IncRNAs. Therefore, we conducted this study using Pearson's method to obtain 630 IncRNAs co-expressed with m6A genes based on the expression levels of 15 m6A genes.

We performed the univariate COX analysis to screen out 21 IncRNAs associated with prognosis and draw forest maps and heat maps. The results showed that 20 IncRNAs are risk factors for prognosis, and one IncRNA is a protective factor for prognosis. According to the expression of 21 IncRNAs, TCGA-THCA cohert were divided into two cluster by consensus clustering method. The results show that patients in cluster2 have a worse prognosis, with higher expression of 21 IncRNAs.

To further study the function of these IncRNAs, we analyzed fractions of infiltrating immune cells between two clusters by CIBERSORT, and the results showed that the content of CD8 T cells, CD4 memory active $T$ cells, follicular helper $T$ cells, macrophages $M 1$, and dendritic cells activated were statistically different, indicating that the immune infiltration between the two clusters was different. Therefore, the ESTIMATE analysis method was used to further explore the tumor microenvironment between the two clusters. Cluster 2 was also found to show a lower estimate score, matrix score, and immune score, which emphasized the role of these IncRNAs in the TME. Considering the importance of PD-L1 during the tumor immunity process [39-42], a correlation between PD-L1 and 21 IncRNAs was observed. The results showed that PD-L1 was negatively correlated with several IncRNAs, and most of the IncRNAs are positively related.

Two datasets (a training group and a testing group) were created by a random method and obtained a prognostic model containing three m6A-related IncRNAs (PSMG3-AS1, BHLHE40-AS1, and AC016747.3) by the LASSO regression analysis in the training group, all of which can be used to further increase the feasibility of our prediction.

Previous reports have indicated that PSMG3-AS1 sponging miR-613 to upregulate SphK1 increases the growth of non-small cell lung cancer cells [43]; and sponging miR-143-3p to increase the proliferation and migration of breast cancer cells [44]; and can function on the migration, proliferation, and invasion of cervical squamous cell carcinoma [45], lung adenocarcinoma [46], hepatocellular carcinoma [47], renal cell carcinoma [48], and breast cancer cells; can distinguish glioblastoma and sarcoidosis [49]; and can potentially predict breast cancer [50] and ovarian cancer [51]. Some studies have shown that early breast cancer progresses by increasingly expressed BHLHE40-AS1 functioning on IL-6/STAT3 signaling [52]. 
AC016747.3 has not been reported, and these three IncRNAs have not been investigated in PTC; therefore, follow-up in vivo and in vitro experiments on these IncRNAs in PTC is necessary.

Furthermore, the prognostic model was verified in the entire TCGA-THCA dataset and the testing group. Based on the prognostic model, the risk score of patients was calculated. According to the median risk value, we divided patients with PTC into high- and low-risk groups. Our analytical results show that patients from high-risk groups have worse PFS than low-risk groups (training dataset: $p=0.004$; testing dataset: $p=0.001$; TCGA-THCA: $p<0.001$ ); ROC curve, PCA, and t-SNE analysis displayed that the prognostic model can be used to predict the patient's PFS to a certain extent. We got an analytical result according to the univariate and multivariate COX analyses: risk score is a prognostic factor independent of other clinical traits for patients with PTC. In the stratified analysis based on clinicopathological characteristics, the prognosis of patients in different groups is also statistically different. The analytical results also showed that some significant difference was observed in the risk scores among several clinicopathological characteristics (RAS, BRAF, N, age, immune score, and cluster) and is correlated with three types of immune cells (resting dendritic cell, resting mast cell, and regulatory $T$ cells). These results increase our understanding of the function of m6A-related IncRNAs in PTC and provide directions for further experiments. To further expand the clinical value of our study, we developed a nomogram to predict the PFS of patients with PTC, performed a drug sensitivity analysis among these three IncRNAs, and found that with the expression of two IncRNAs increased sensitivity or resistance to certain chemotherapeutic drugs. Notably, as the expression level of these two IncRNAs increases, the sensitivity of tumor cells to mitoxantrone increases. Moreover, these three IncRNAs also have a certain prognostic value in pan-cancer.

This study has some limitations that should be considered. First, it is the data source from the TCGATHCA cohort used to analyze and verify the prognostic significance of m6A-related IncRNAs. To further verify the prognostic model, we still lack more central data. Second, only the correlation between the expression of these three IncRNAs and clinicopathological characteristics, immune checkpoints, drug sensitivity, or pan-cancer was considered; however, we did not analyze pathways and mechanisms of their effects in in vivo and in vitro experiments.

\section{Conclusion}

In summary, m6A-related IncRNAs of patients with PTC were obtained based on public databases and constructed and verified a prognostic model. The three m6A-related IncRNAs in the prognostic model can be used as prognostic markers for patients with PTC. In addition, the functional analysis also provides a new perspective in future studies on the role and mechanism of m6A-related IncRNAs in patients with PTC.

\section{Abbreviations}


LncRNAs: long non-coding RNAs; PTC: papillary thyroid carcinoma; LASSO: least absolute shrinkage and selection operator; TCGA: The Cancer Genome Atlas database; PD-L1:programmed death-ligand 1, TME: tumor microenvironment; TMB: tumor mutation burden; m6A:N (6)-methyladenosine; mRNA: messenger RNA; UCSC: University of California Santa Cruz; PFS: progression-free survival; ESTIMATE: Estimation of Stromal and Immune cells in Malignant Tumor tissues using Expression data; AUC: area under the curve; ROC: receiver operating characteristic; PCA: principal component analysis; t-SNE: t-Distributed Stochastic Neighbor Embedding; AJCC: American Joint Committee on Cancer; GEPIA2: Gene Expression Profiling Interactive Analysis 2; GTEx:Genotype-Tissue Expression Project database; HR: hazard ratio; Cl: confidence interval.

\section{Declarations}

\section{Acknowledgements}

We appreciate the TCGA database for allowing access to their data.

\section{Authors' contributions}

$Y D H$ created the analytical strategies and drafted the paper. $X L$ verified the data analysis method. $X Y L$, $\mathrm{YL}, \mathrm{XL}, \mathrm{YZH}, \mathrm{SW}, \mathrm{SYW}, \mathrm{YCH}$, and HJ performed data analyses. JZ designed and wrote the paper. Each author equally contributed to the article, and all of them approved the final draft that was eventually published.

\section{Funding}

The National Natural Science Foundation of China sponsored this study (81600602).

\section{Availability of data and materials}

The data of current study are available from TCGA dataset.

\section{Declarations}

\section{Ethics approval and consent to participate}

Not applicable.

\section{Consent for publication}

The authors agree for publication. 


\section{Competing interests}

The writers indicated that their work was not influenced by competing financial interests or personal connections.

\section{References}

1. Pellegriti G, Frasca F, Regalbuto C, Squatrito S, Vigneri R (2013) Worldwide increasing incidence of thyroid cancer: update on epidemiology and risk factors. J Cancer Epidemiol 2013:965212

2. Rajabi S, Dehghan MH, Dastmalchi R, Jalali Mashayekhi F, Salami S, Hedayati M (2019) The roles and role-players in thyroid cancer angiogenesis. Endocr J 66:277-293

3. Schneider DF, Chen H (2013) New developments in the diagnosis and treatment of thyroid cancer. CA Cancer J Clin 63:374-394

4. Fagin JA, Wells SA (2016) Jr.: Biologic and Clinical Perspectives on Thyroid Cancer. N Engl J Med 375:1054-1067

5. Xing M (2013) Molecular pathogenesis and mechanisms of thyroid cancer. Nat Rev Cancer 13:184199

6. Lamartina L, Grani G, Durante C, Filetti S (2018) Recent advances in managing differentiated thyroid cancer. F1000Res 7:86

7. Davies L, Morris LG, Haymart M, Chen AY, Goldenberg D, Morris J, Ogilvie JB, Terris DJ, Netterville J, Wong RJ, Randolph G (2015) AMERICAN ASSOCIATION OF CLINICAL ENDOCRINOLOGISTS AND AMERICAN COLLEGE OF ENDOCRINOLOGY DISEASE STATE CLINICAL REVIEW: THE INCREASING INCIDENCE OF THYROID CANCER. Endocr Pract 21:686-696

8. He L, Li H, Wu A, Peng Y, Shu G, Yin G (2019) Functions of N6-methyladenosine and its role in cancer. Mol Cancer 18:176

9. Wang X, Lu Z, Gomez A, Hon GC, Yue Y, Han D, Fu Y, Parisien M, Dai Q, Jia G et al (2014) N6methyladenosine-dependent regulation of messenger RNA stability. Nature 505:117-120

10. Deng X, Su R, Weng H, Huang H, Li Z, Chen J (2018) RNA N(6)-methyladenosine modification in cancers: current status and perspectives. Cell Res 28:507-517

11. Panneerdoss S, Eedunuri VK, Yadav P, Timilsina S, Rajamanickam S, Viswanadhapalli S, Abdelfattah $\mathrm{N}$, Onyeagucha BC, Cui X, Lai Z et al (2018) Cross-talk among writers, readers, and erasers of $\mathrm{m}(6) \mathrm{A}$ regulates cancer growth and progression. Sci Adv 4:eaar8263

12. Ramilowski JA, Yip CW, Agrawal S, Chang JC, Ciani Y, Kulakovskiy IV, Mendez M, Ooi JLC, Ouyang JF, Parkinson $\mathrm{N}$ et al (2020) Functional annotation of human long noncoding RNAs via molecular phenotyping. Genome Res 30:1060-1072

13. Huang Q, Yan J, Agami R (2018) Long non-coding RNAs in metastasis. Cancer Metastasis Rev $37: 75-81$ 
14. Feng ZY, Gao HY, Feng TD (2021) Immune Infiltrates of m(6)A RNA Methylation-Related IncRNAs and Identification of PD-L1 in Patients With Primary Head and Neck Squamous Cell Carcinoma. Front Cell Dev Biol 9:672248

15. Cancer Genome Atlas Research Network, Weinstein JN, Collisson EA, Mills GB, Shaw KR, Ozenberger BA, Ellrott K, Shmulevich I, Sander C, Stuart JM. The Cancer Genome Atlas Pan-Cancer analysis project. Nat Genet. 2013 Oct;45(10):1113-1120. doi:10.1038/ng.2764. PMID: 24071849; PMCID: PMC3919969

16. Goldman MJ, Craft B, Hastie M, Repečka K, McDade F, Kamath A, Banerjee A, Luo Y, Rogers D, Brooks AN et al (2020) Visualizing and interpreting cancer genomics data via the Xena platform. Nat Biotechnol 38:675-678

17. Haeussler M, Zweig AS, Tyner C, Speir ML, Rosenbloom KR, Raney BJ, Lee CM, Lee BT, Hinrichs AS, Gonzalez JN et al (2019) The UCSC Genome Browser database: 2019 update. Nucleic Acids Res 47:D853-Dd858

18. Tang J, Wan Q, Lu J (2020) The prognostic values of m6A RNA methylation regulators in uveal melanoma. BMC Cancer 20:674

19. Tian S, Lai J, Yu T, Li Q, Chen Q (2020) Regulation of Gene Expression Associated With the N6Methyladenosine (m6A) Enzyme System and Its Significance in Cancer. Front Oncol 10:623634

20. He PC, He C (2021) m(6) A RNA methylation: from mechanisms to therapeutic potential. Embo j 40:e105977

21. Friedman J, Hastie T, Tibshirani R (2010) Regularization Paths for Generalized Linear Models via Coordinate Descent. J Stat Softw 33:1-22

22. Huang R, Li G, Wang Z, Hu H, Zeng F, Zhang K, Wang K, Wu F (2020) Identification of an ATP metabolism-related signature associated with prognosis and immune microenvironment in gliomas. Cancer Sci 111:2325-2335

23. Tang Z, Kang B, Li C, Chen T, Zhang Z (2019) GEPIA2: an enhanced web server for large-scale expression profiling and interactive analysis. Nucleic Acids Res 47:W556-Ww560

24. Liu X, Wang P, Teng X, Zhang Z, Song S (2021) Comprehensive Analysis of Expression Regulation for RNA m6A Regulators With Clinical Significance in Human Cancers. Front Oncol 11:624395

25. He J, Zhou M, Yin J, Wan J, Chu J, Jia J, Sheng J, Wang C, Yin H, He F (2021) METTL3 restrains papillary thyroid cancer progression via $\mathrm{m}(6) \mathrm{A} / \mathrm{c}-\mathrm{Rel} / \mathrm{IL}-8-$-mediated neutrophil infiltration. Mol Ther 29:1821-1837

26. Wang K, Jiang L, Zhang Y, Chen C (2020) Progression of Thyroid Carcinoma Is Promoted by the m6A Methyltransferase METTL3 Through Regulating m(6)A Methylation on TCF1. Onco Targets Ther 13:1605-1612

27. Tian R, Zhang S, Sun D, Bei C, Li D, Zheng C, Song X, Chen M, Tan S, Zhu X, Zhang H: M6A Demethylase FTO Plays a Tumor Suppressor Role in Thyroid Cancer. DNA Cell Bio/ 2020

28. Hou J, Shan H, Zhang Y, Fan Y, Wu B (2020) m(6)A RNA methylation regulators have prognostic value in papillary thyroid carcinoma. Am J Otolaryngol 41:102547 
29. Wang X, Fu X, Zhang J, Xiong C, Zhang S, Lv Y (2020) Identification and validation of m(6)A RNA methylation regulators with clinical prognostic value in Papillary thyroid cancer. Cancer Cell Int 20:203

30. Xu N, Chen J, He G, Gao L, Zhang D (2020) Prognostic values of m6A RNA methylation regulators in differentiated Thyroid Carcinoma. J Cancer 11:5187-5197

31. Lan Y, Liu B, Guo H (2021) The role of M(6)A modification in the regulation of tumor-related IncRNAs. Mol Ther Nucleic Acids 24:768-779

32. Yuan Q, Ren J, Li L, Li S, Xiang K, Shang D (2021) Development and validation of a novel N6methyladenosine (m6A)-related multi- long non-coding RNA (IncRNA) prognostic signature in pancreatic adenocarcinoma. Bioengineered 12:2432-2448

33. Zheng J, Guo J, Cao B, Zhou Y, Tong J (2021) Identification and validation of IncRNAs involved in m6A regulation for patients with ovarian cancer. Cancer Cell Int 21:363

34. Li L, Xie R, Lu G: Identification of m6A methyltransferase-related IncRNA signature for predicting immunotherapy and prognosis in patients with hepatocellular carcinoma. Biosci Rep 2021, 41

35. Wu J, Cai Y, Zhao G, Li M (2021) A ten N6-methyladenosine-related long non-coding RNAs signature predicts prognosis of triple-negative breast cancer. J Clin Lab Anal 35:e23779

36. Yu J, Mao W, Sun S, Hu Q, Wang C, Xu Z, Liu R, Chen S, Xu B, Chen M (2021) Identification of an m6A-Related IncRNA Signature for Predicting the Prognosis in Patients With Kidney Renal Clear Cell Carcinoma. Front Oncol 11:663263

37. Huang S, Lyu S, Gao Z, Zha W, Wang P, Shan Y, He J, Li Y (2021) m6A-Related IncRNAs Are Potential Biomarkers for the Prognosis of Metastatic Skin Cutaneous Melanoma. Front Mol Biosci 8:687760

38. Tu Z, Wu L, Wang P, Hu Q, Tao C, Li K, Huang K, Zhu X (2020) N6-Methylandenosine-Related IncRNAs Are Potential Biomarkers for Predicting the Overall Survival of Lower-Grade Glioma Patients. Front Cell Dev Biol 8:642

39. Ishida Y, Agata Y, Shibahara K, Honjo T (1992) Induced expression of PD-1, a novel member of the immunoglobulin gene superfamily, upon programmed cell death. Embo j 11:3887-3895

40. Francisco LM, Sage PT, Sharpe AH (2010) The PD-1 pathway in tolerance and autoimmunity. Immunol Rev 236:219-242

41. Asano T, Meguri Y, Yoshioka T, Kishi Y, Iwamoto M, Nakamura M, Sando Y, Yagita H, Koreth J, Kim HT et al (2017) PD-1 modulates regulatory T-cell homeostasis during low-dose interleukin-2 therapy. Blood 129:2186-2197

42. Sun Y, Tan J, Miao Y, Zhang Q (2021) The role of PD-L1 in the immune dysfunction that mediates hypoxia-induced multiple organ injury. Cell Commun Signal 19:76

43. Shen W, Hong $X$, Jin C, Xi Y (2021) LncRNA PSMG3AS1 promotes proliferation of non-small cell lung cancer cells by sponging miR-613 to upregulate SphK1. Cell Cycle 20:829-838

44. Cui Y, Fan Y, Zhao G, Zhang Q, Bao Y, Cui Y, Ye Z, Chen G, Piao X, Guo F et al (2020) Novel IncRNA PSMG3-AS1 functions as a miR-143-3p sponge to increase the proliferation and migration of 
breast cancer cells. Oncol Rep 43:229-239

45. Man S, Li X, Zhu W (2021) miR-4417 targets IncRNA PSMG3-AS1 to suppress cell invasion and migration in cervical squamous cell carcinoma. Oncol Lett 22:502

46. Yue N, Ye M, Zhang R, Guo Y (2020) MiR-449b-5p targets IncRNA PSMG3-AS1 to suppress cancer cell proliferation in lung adenocarcinoma. BMC Pulm Med 20:152

47. Zhang J, Huang J, Chen W, Hu Z, Wang X (2020) miR-143-3p Targets IncRNA PSMG3-AS1 to Inhibit the Proliferation of Hepatocellular Carcinoma Cells. Cancer Manag Res 12:6303-6309

48. Cheng G, Liu D, Liang H, Yang H, Chen K, Zhang X (2019) A cluster of long non-coding RNAs exhibit diagnostic and prognostic values in renal cell carcinoma. Aging 11:9597-9615

49. Chen L, Wang G, Xu Z, Lin K, Mu S, Pan Y, Shan M (2020) Overexpression of LncRNA PSMG3-AS1 Distinguishes Glioblastomas from Sarcoidosis. J Mol Neurosci 70:2015-2019

50. Gong PJ, Shao YC, Huang SR, Zeng YF, Yuan XN, Xu JJ, Yin WN, Wei L, Zhang JW (2020) HypoxiaAssociated Prognostic Markers and Competing Endogenous RNA Co-Expression Networks in Breast Cancer. Front Oncol 10:579868

51. Li N, Zhan X (2019) Identification of clinical trait-related IncRNA and mRNA biomarkers with weighted gene co-expression network analysis as useful tool for personalized medicine in ovarian cancer. Epma j 10:273-290

52. DeVaux RS, Ropri AS, Grimm SL, Hall PA, Herrera EO, Chittur SV, Smith WP, Coarfa C, Behbod F, Herschkowitz JI (2020) Long noncoding RNA BHLHE40-AS1 promotes early breast cancer progression through modulating IL-6/STAT3 signaling. J Cell Biochem 121:3465-3478

\section{Figures}




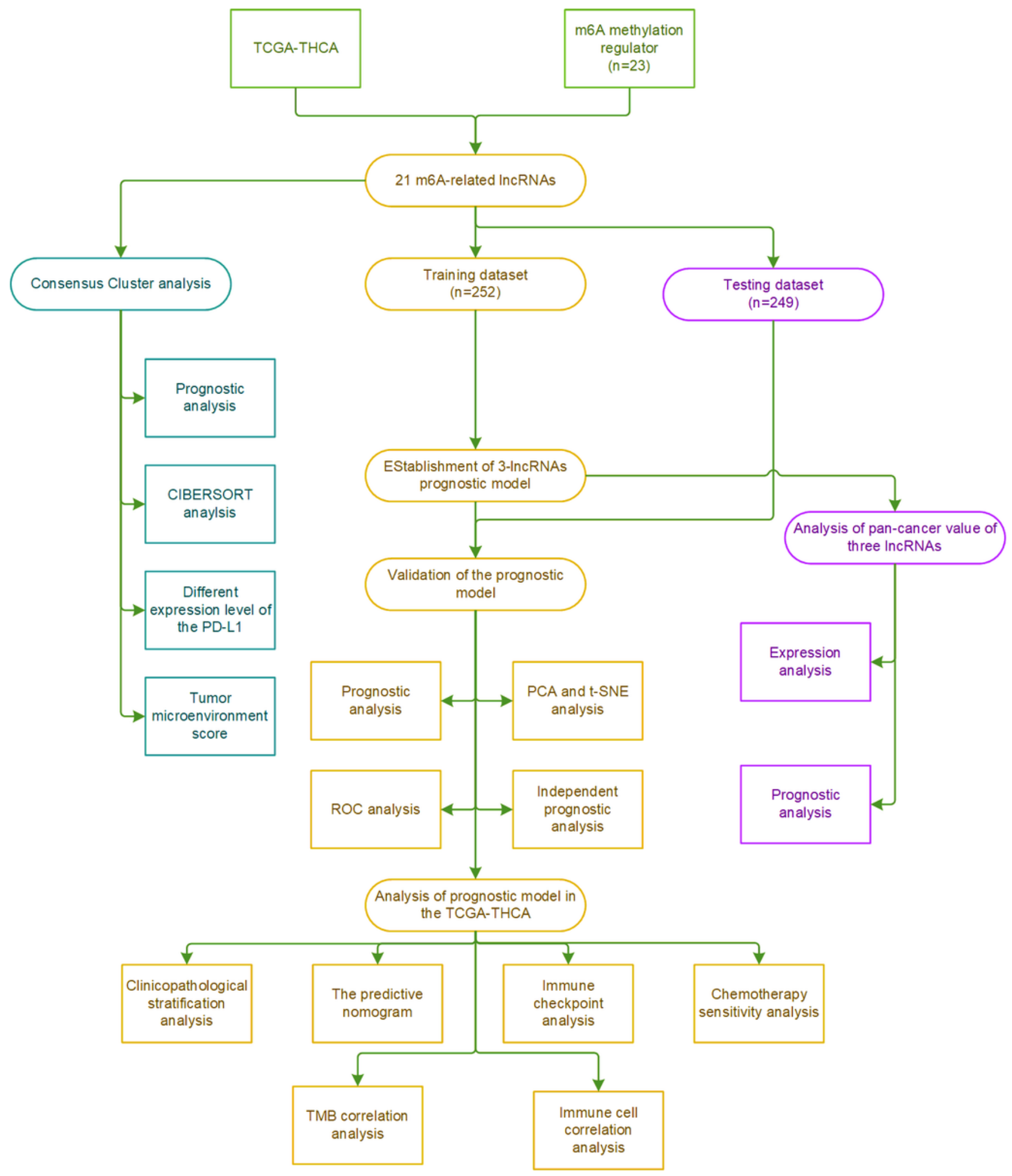

Figure 1

Data analysis flowchart. 

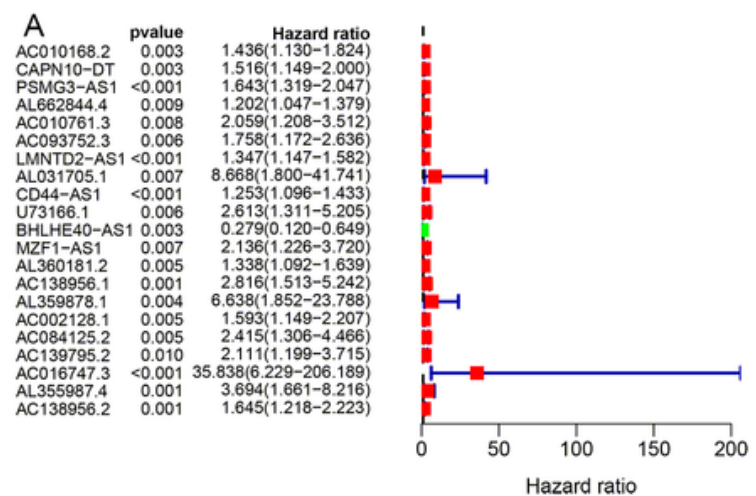

B

Type 白 Normal 追 Tumor
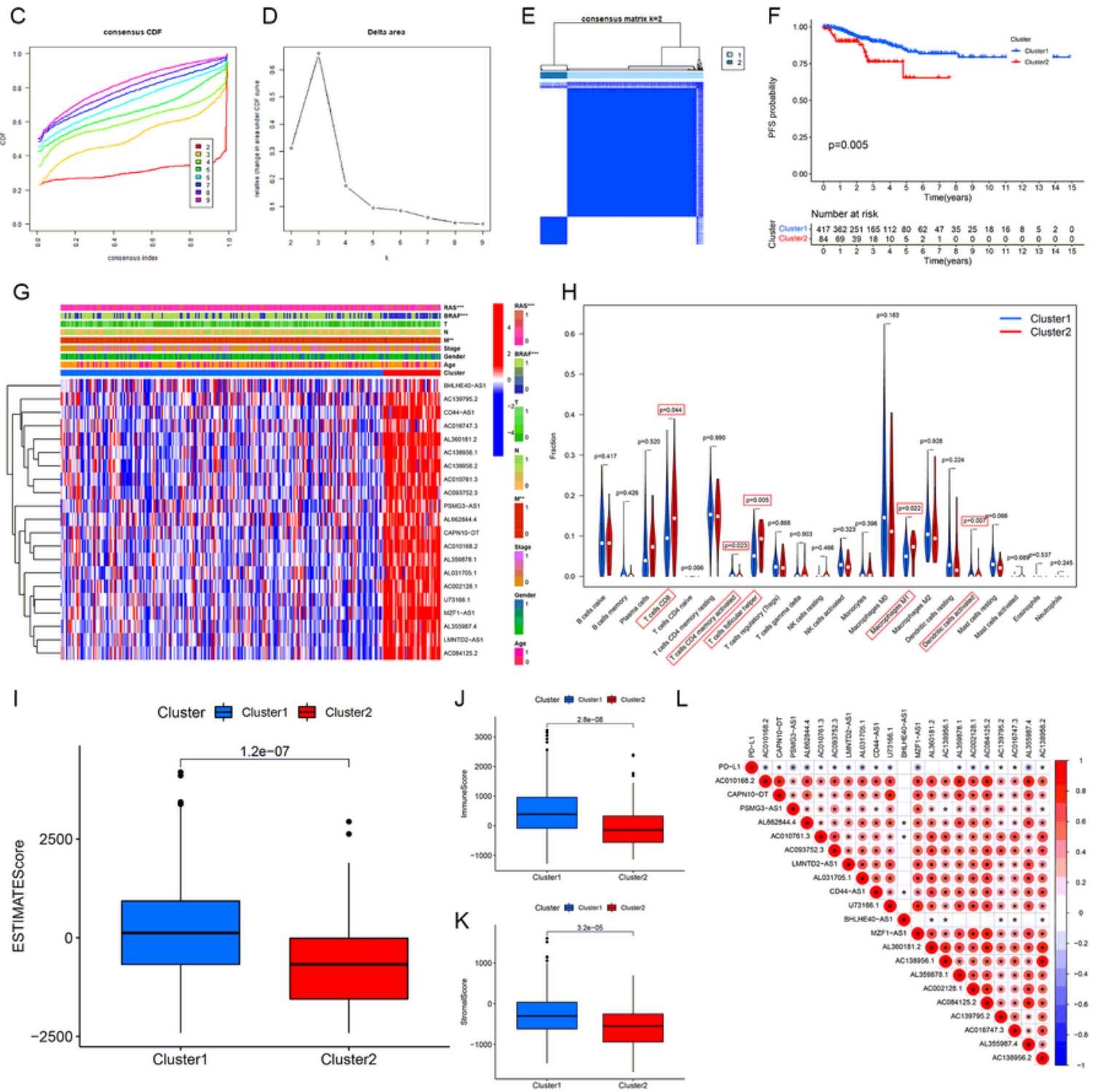

Figure 2

Consensus cluster analysis. (A) The forest plot of differentially expressed IncRNAs for prognosis; (B) The box plot of differentially expressed IncRNAs for prognosis; (C) Consensus CDF curve; (D) A relative increase in the CDF graph area under the curve for $\mathrm{k}=2-9$; (E) The TCGA-THCA dataset was divided into cluster 1 and cluster 2 with $k=2$; $(F)$ Progression-free survival probability between the two clusters; $(G)$ Heatmap of clinicopathological characteristics between two clusters; $(\mathrm{H})$ Violin plot of immune cell 
infiltration between two clusters; (I) Box plot of ESTIMATE scores between two clusters; $(\mathrm{J})$ Box plot of Immune scores between two clusters; (K) Box plot of Stromal scores between two clusters; (L) Correlation between PD-L1 and 21 IncRNAs.
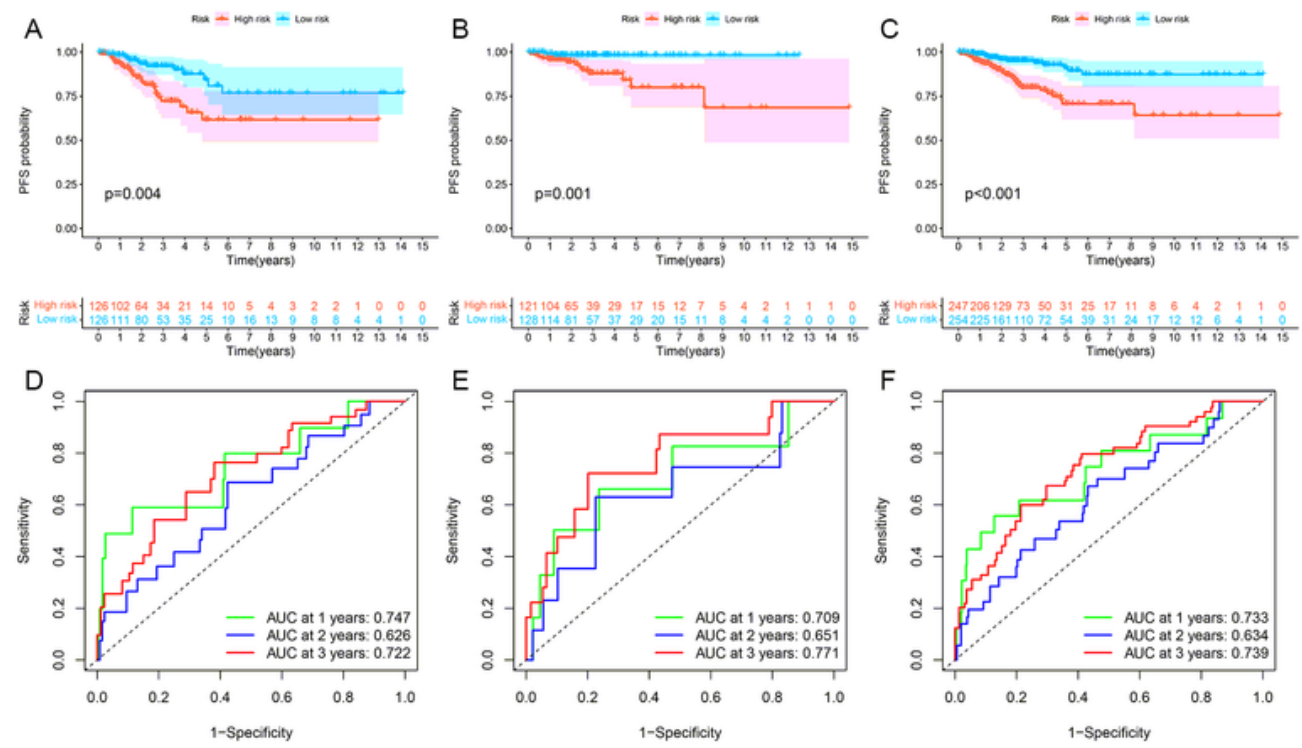

G

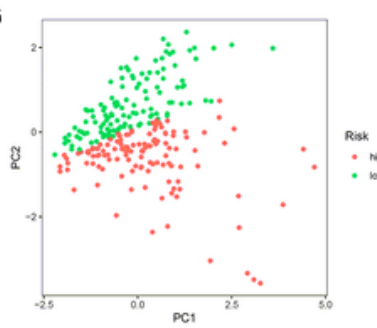

$J$
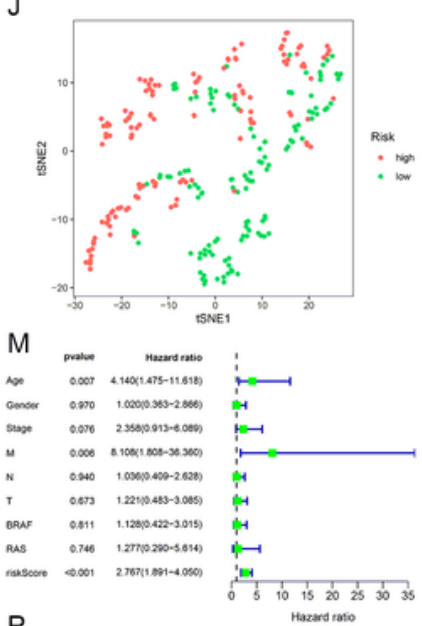

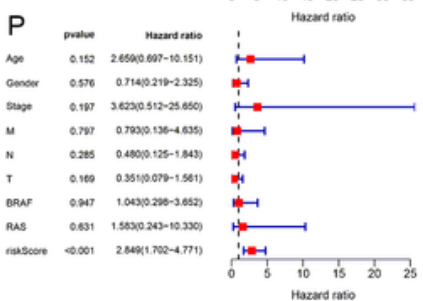

$\mathrm{H}$

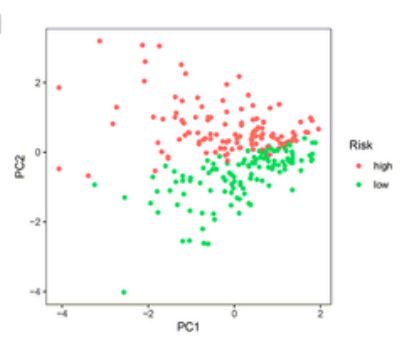

K
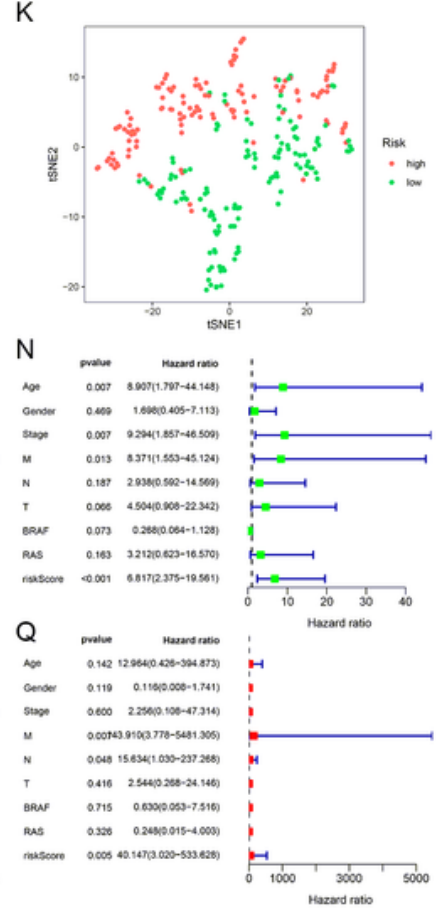
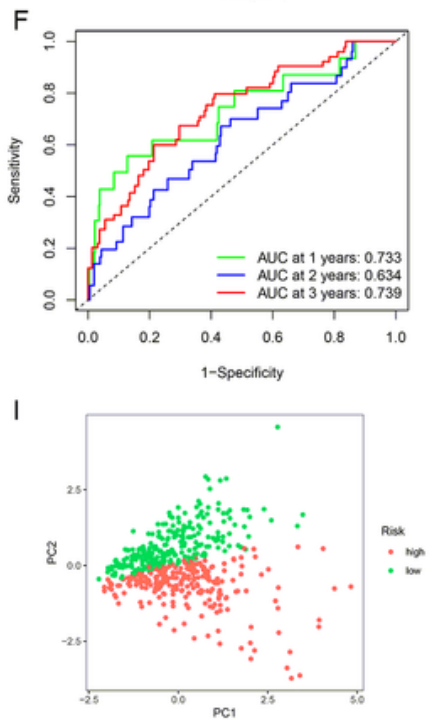

L

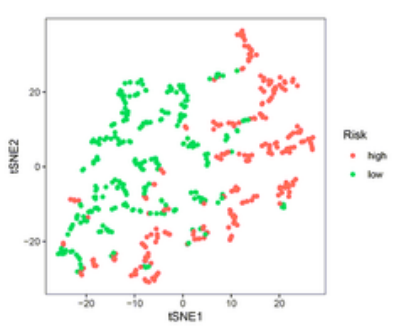

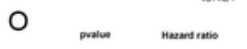
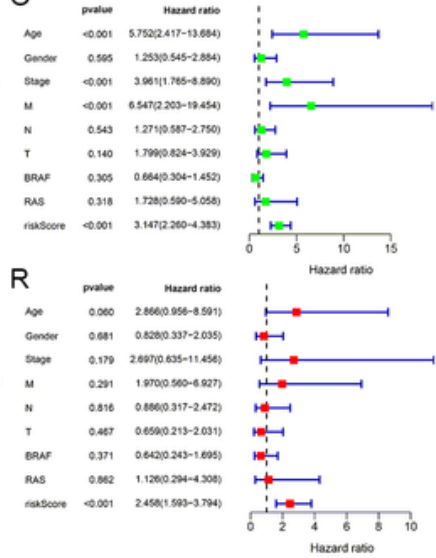

Figure 3

(A) The K-M curve for PFS between the high- and low-risk groups in the training dataset. (B) The K-M curve for PFS between the high- and low-risk groups in the testing dataset. (C) K-M curve for PFS between 
the high- and low-risk groups in the TCGA-THCA dataset. (D) ROC curve for predicting PFS in the training dataset. (E) ROC curve to predict the PFS in the testing dataset. (F) ROC curve to predict PFS in the TCGATHCA dataset. (G) PCA plot in the training dataset. $(\mathrm{H})$ PCA plot in the testing dataset. (I) PCA plot in the TCGA-THCA dataset. $(\mathrm{J}) \mathrm{t}$-SNE analysis of the training dataset. $(\mathrm{K}) \mathrm{t}-\mathrm{SNE}$ analysis of the testing dataset. (L) t-SNE analysis of the TCGA-THCA dataset. (M-R) Results of univariate and multivariate cox analyses indicate that the risk score is an independent prognostic indicator for patients with PTC. (M) Univariate cox analysis in the training dataset. $(\mathrm{N})$ Univariate cox analysis in the training dataset. (O) Univariate cox analysis in the TCGA-THCA dataset. (P) Multivariate cox analysis in the training dataset. (Q) Multivariate cox analysis in the testing dataset. (R) Multivariate cox analysis in the TCGA-THCA dataset.
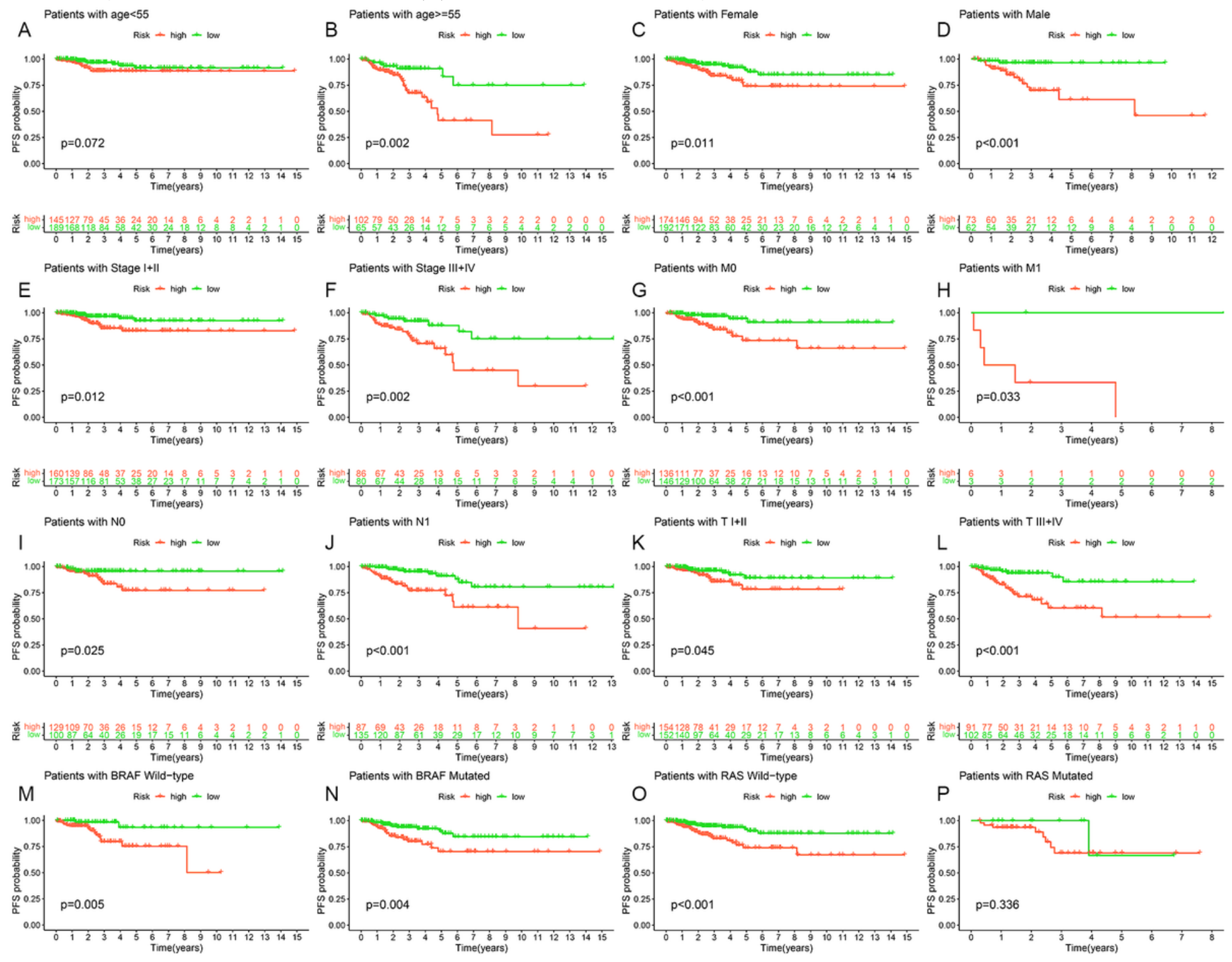

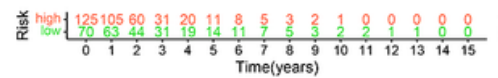
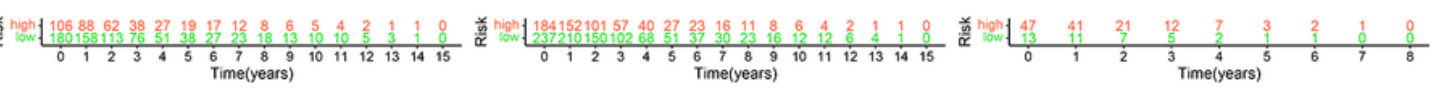

\section{Figure 4}

Stratified analysis of patient prognosis based on risk scores in different clinicopathological characteristics in the TCGA-THCA dataset. (A, B) Age ( $<55$ vs. $\geq 55$ years). (C, D) Gender (female vs. 
male). (E, F) Stage (stage I+II vs. III+IV). (G, H) M (M0 vs. M1). (I, J) N (N0 vs. N1). (K, L) T (T I+II vs. III+IV). $(\mathrm{M}, \mathrm{N})$ BRAF (wild type vs. mutated). (O, P) RAS (wild type vs. mutated).
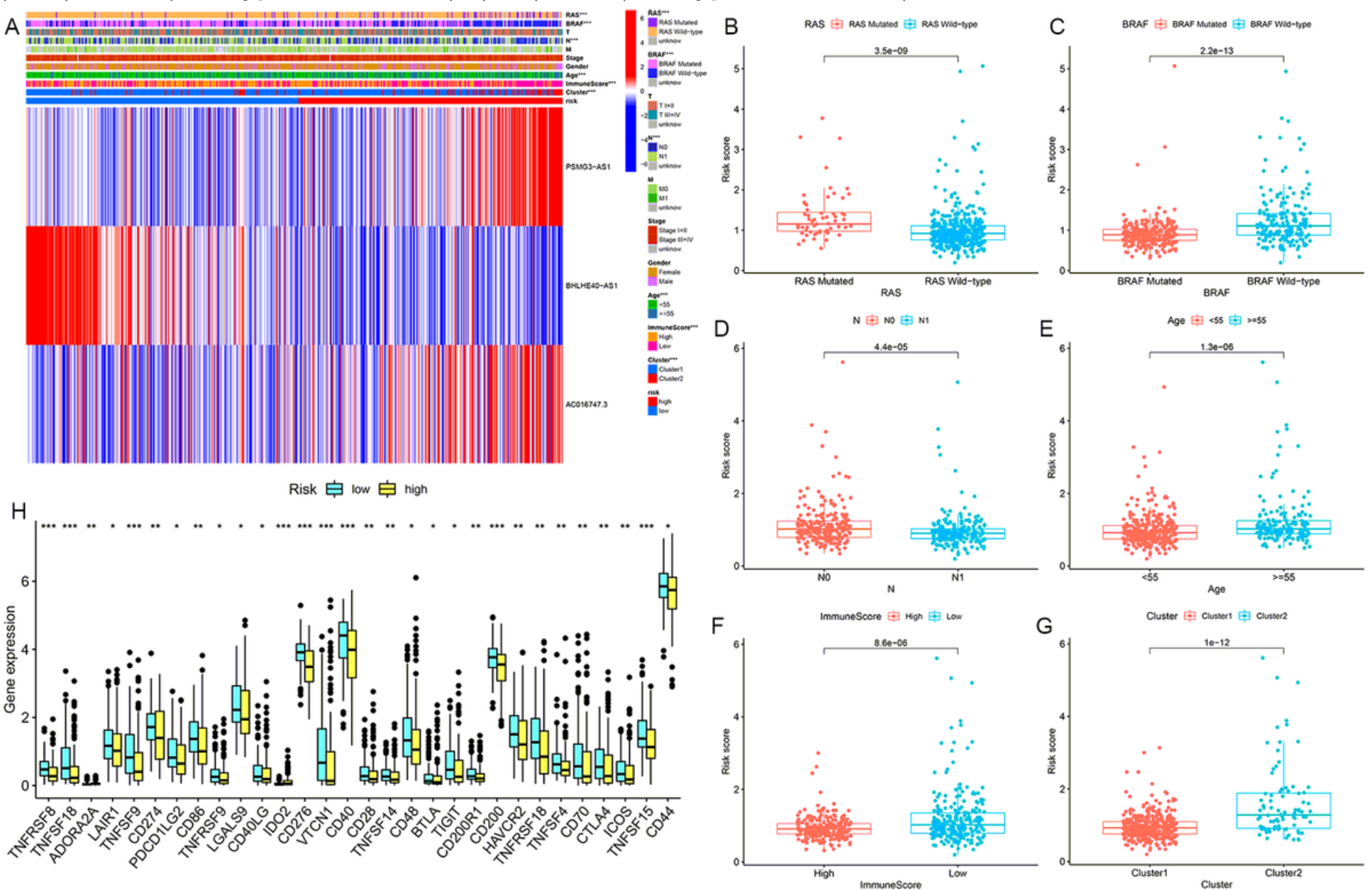

Figure 5

Correlation between risk scores and clinicopathological characteristics, immune scores, and immune checkpoints in the TCGA-THCA dataset. (A) Heatmap of the expression of m6A-related IncRNAs in different risk groups and clinicopathological characteristics. (B-G) Box plot of risk scores classified according to clinicopathological characteristics and immune score. $(H)$ Expression of immune checkpoints between high- and low-risk groups. 

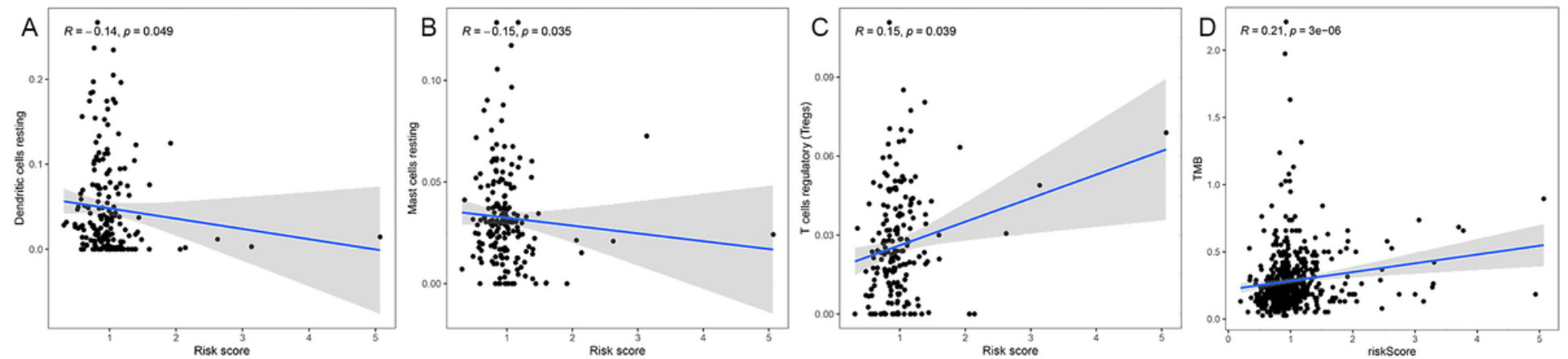

E

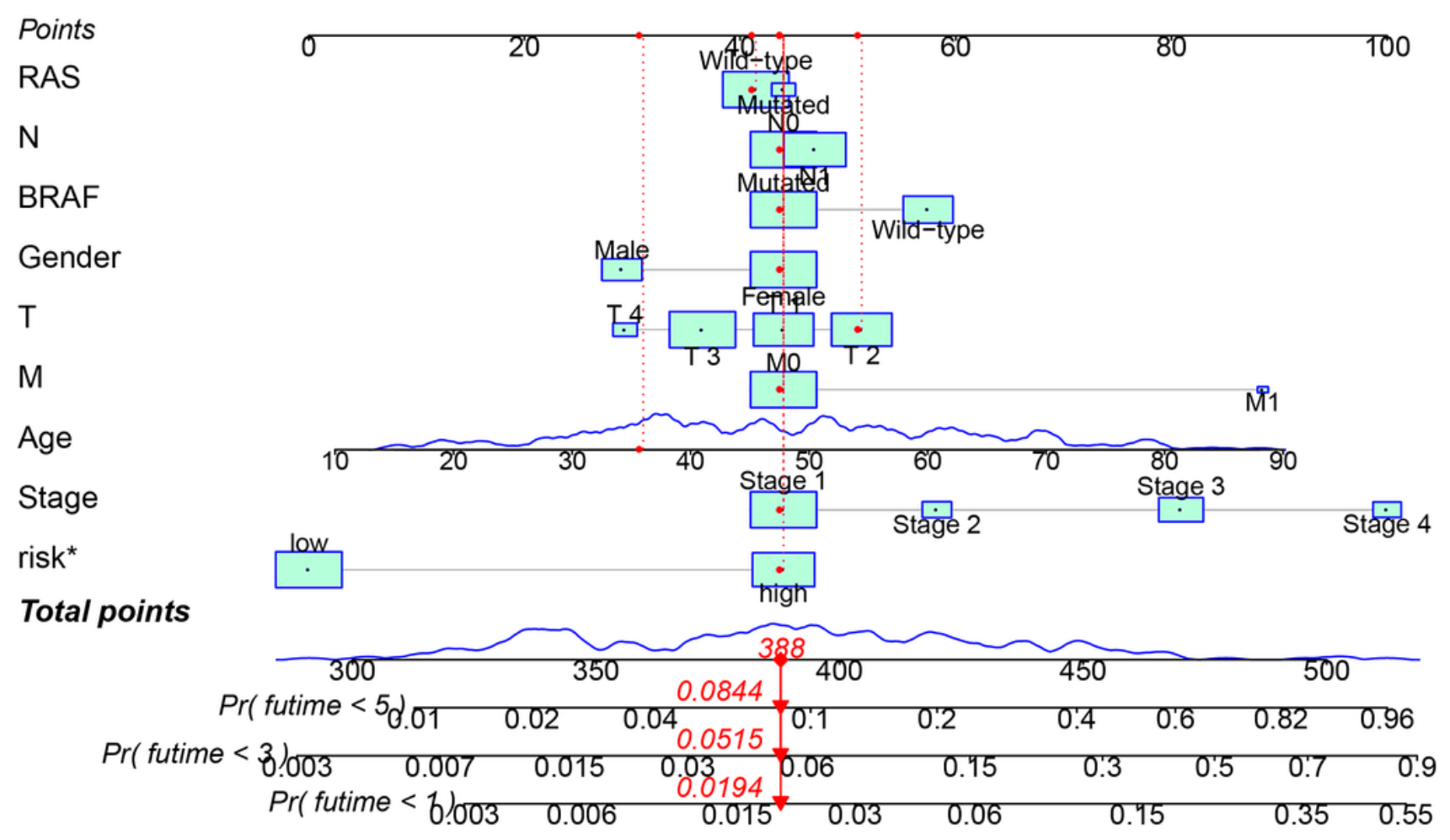

Figure 6

Correlation analysis of the risk score, immune cells, and tumor mutation burden, and construction of the nomogram. Correlation between risk score and resting dendritic cells (A), resting mast cells (B), regulatory T cells (C), and TMB (D). (E) A nomogram was constructed based on risk scores and clinicopathological characteristics to predict the progression-free survival of patients with PTC at 1, 3, and 5 years. 
A PSMG3-AS1, Mitomycin

Cor $=0.384, p=0.002$

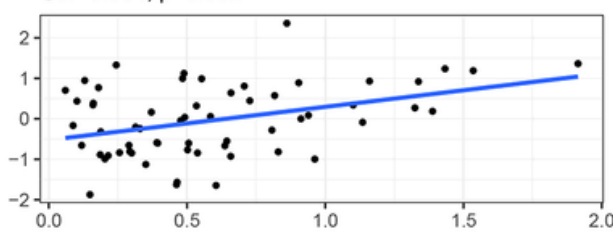

PSMG3-AS1, Tegafur

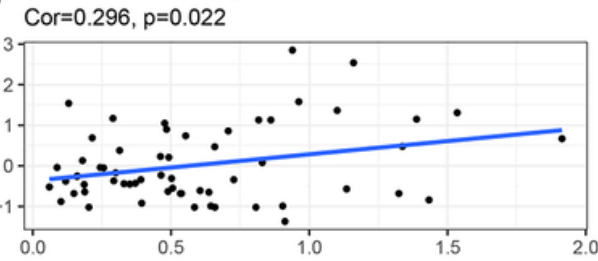

BHLHE40-AS1, Epirubicin

$G$ Cor $=0.261, p=0.044$

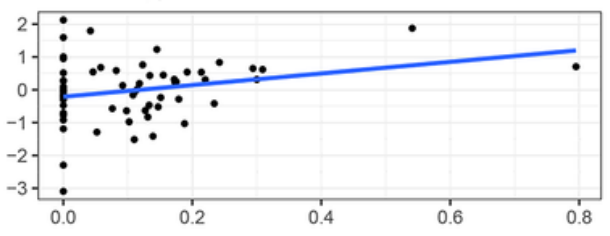

J BHLHE40-AS1, Doxorubicin

Cor $=0.259, p=0.045$

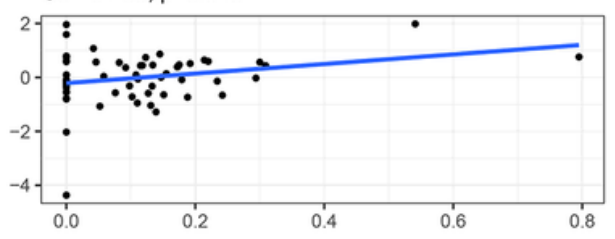

BHLHE40-AS1, Allopurinol

Cor $=-0.309, p=0.016$

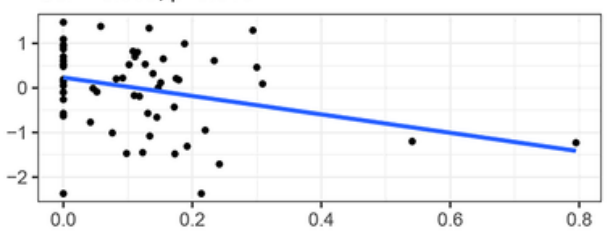

E PSMG3-AS1, Idarubicin

Cor $=0.295, p=0.022$

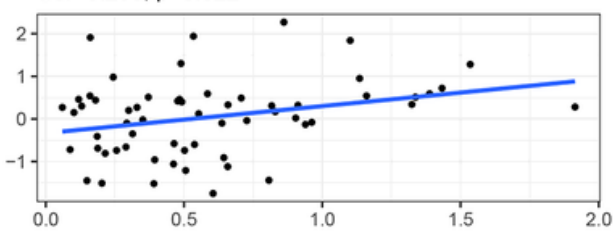

BHLHE40-AS1, MITOXANTRONE

H Cor $=0.261, p=0.044$

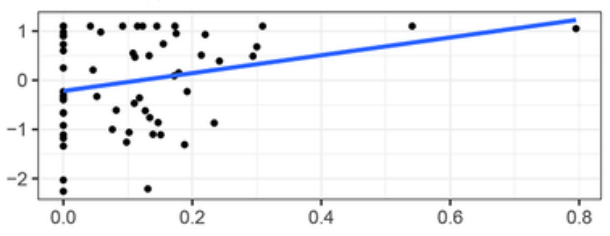

K PSMG3-AS1, Bosutinib

K Cor $=-0.258, p=0.046$

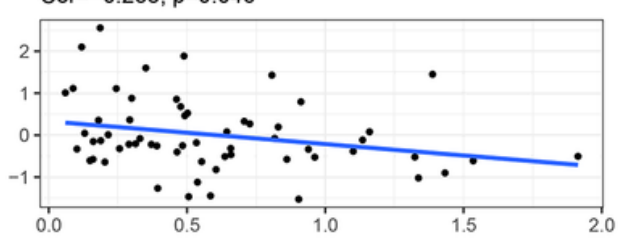

C PSMG3-AS1, MITOXANTRONE

Cor $=0.306, p=0.018$

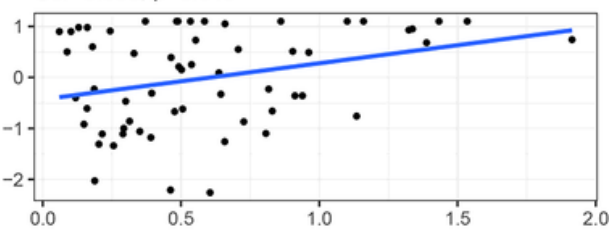

F PSMG3-AS1, Gemcitabine

Cor $=0.266, p=0.040$

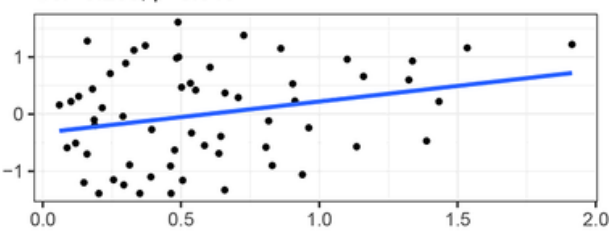

PSMG3-AS1, Fulvestrant

Cor $=0.260, p=0.044$

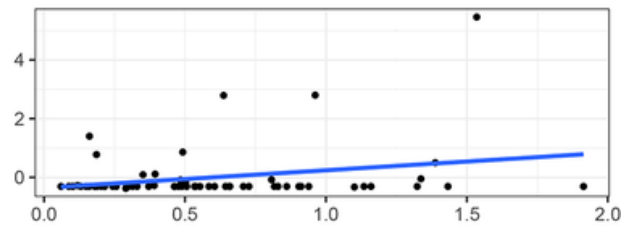

PSMG3-AS1, Teniposide

$L$ Cor $=0.255, p=0.049$

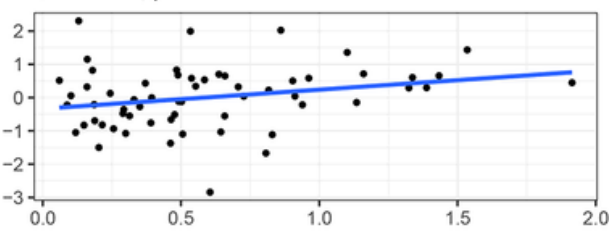

Figure 7

Scatter plot of m6A-related IncRNAs expression and drug sensitivity. 

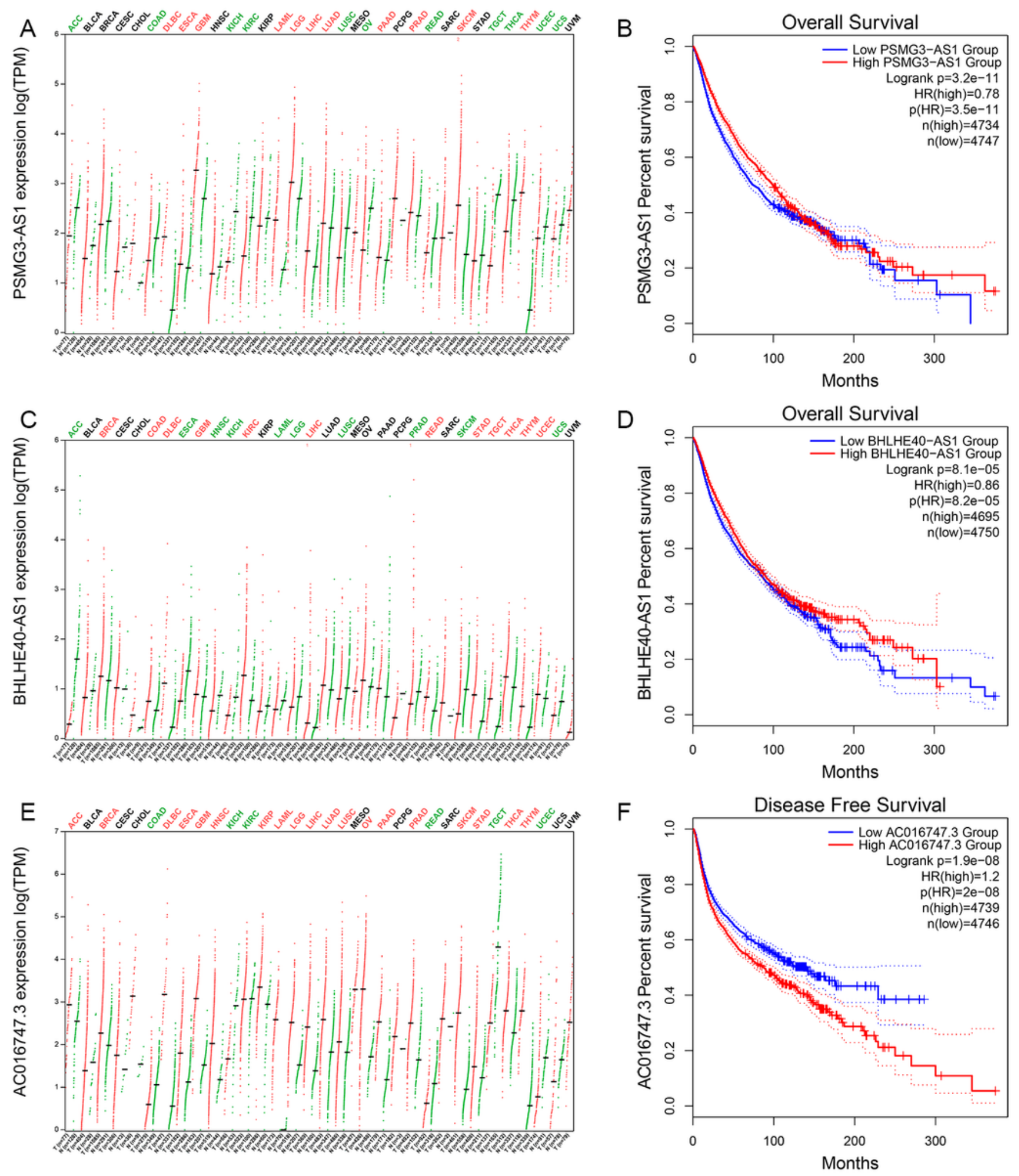

\section{Figure 8}

The value of m6A-related IncRNAs in pan-cancer. (A) Comparison of PSMG3-AS1 expression in 33 cancers and paracancerous tissues based on GEPIA2. (B) Comparing the overall survival (OS) rate of high- and low-PSMG3-AS1 groups in 33 cancers based on GEPIA2. (C) Comparison of BHLHE40-AS1 expression in 33 cancers and paracancerous tissues based on GEPIA2. (D) Comparing the OS rate of high- and low-BHLHE40-AS1 groups in 33 cancers based on GEPIA2. (E) Comparison of AC016747.3 
expression in 33 cancers and paracancerous tissues based on GEPIA2. (F) Comparing the disease-free survival (RFS) rate of high- and low AC016747.3 groups in 33 cancers based on GEPIA2.

\section{Supplementary Files}

This is a list of supplementary files associated with this preprint. Click to download.

- SupplementaryFigure1.tif

- SupplementaryFigure2.tif

- SupplementaryFigure3.tif

- SupplementaryFigure4.tif

- SupplementaryList1.txt 